\title{
Tendencias y perspectivas de la integración económica en América Latina
}

1. PROYECTOS DE INTEGRACIÓN $Y$ REALIDADES DE INTEGRAGIÓN EN UNA PERSPECTIVA HISTÓRICA

Desde fines de la década del cincuenta los países latinoamericanos se han comprometido formalmente al desarrollo de procesos de integración de sus economías. Los Tratados de Montevideo y de Managua, son las expresiones jurídicas principales de dichos compromisos. Los mismos se han formalizado sobre la base de proyectos o programas de integración, en parte influenciados por los imperativos de la coyuntura internacional, en parte por el efecto de demostración de modelos extrarregionales como el de las Comunidades Europeas, $y$ en parte por la incidencia del pensamiento económico elaborado en organismos regionales preocupados por el desarrollo económico reegional.

En el caso de la ALALC, es sabido que las distintas fuentes de intereses e ideológicas que alimentan el programa original de integración explican el contenido ambiguo del Tratado de Montevideo con respecto a algunos de los objetivos incorporados en el mismo (por ej. el del artículo 54), a la rez que reflejan el conflicto de concepción que a veces en estado latente, en otras en estado manifiesto, ha sacudido la marcha de la Asociación a través de los años. El Tratado y muchas de sus resoluciones posteriores son ejemplos elocuentes de lo que se ha podido denominar tendencia al "cinismo legislativo" y al "utopismo legislativo" en el proceso de creación normativa de la región $n^{x}$ En ambas hipótesis, la resultante es una distancia mayor o menor entre la norma y lo que los comprometidos desean o pueden cumplir. Examinese como ejemplo la historia de la Resolución 100 (IV) y de su efectividad posterior.

Pero, estos programas o proyectos de integración han dado lugar o han sido acompañados, o aun en ocasiones han sido superados, por acciones concretas de integración encaradas por gobiernos y empresas. Acciones que a pesar de su carácter poco sistemático y aun errático, muestran en, su conjunto un progreso significativo en la

ICf. INTAL, "La solución de conflictos en la integración latinoamericana", Serie Estudios, NS 8, 1973. 
formación e institucionalización de un sistema económico y político latinoamericano que ha alcanzaclo ya un grado interesante de interacción entre las unidades que lo componen. Los indicadoies más clásicos de interacción entre los componentes de un determinado subsistema internacional muestran un contraste claro en el caso de América Latina si se compara la situación en 1960 con la existente en 1974. Puede demostrarse con cierta facilidad que los países de la región se consideran recíprocamente como merca:dos valiosos para la proyección externa de sus economías o para el aprovisionamiento de recursos productivos necesarios al desarrollo interno, y como modelos a tener en cuenta al encarar sus propios problemas internos. La región es fuente valorada de mercados y de modelos. Antes, la demanda externa, los recursos productivos y las fuentes de inspiración, eran casi exclusivamente extrazonales. A su vez, la red de canales institucionalizados de comunicación entre los países de la región, desbordan ampliamente Ios de la ALALG, y se manifiestan a veces en marcos multilaterales interamericanos (BDD, OEA) o latinoamericanos (GECLA) o en marcos bilaterales formales o informales, permanentes o circunstanciales (comisiones mixtas, intercambio constante en visitas oficiales, etc.).

Gierto es que tales interacciones no se limitan al plano de la cooperación y la integración, y es a veces en el plano del conflicto, donde las mismas tienden a ser más intensas. En menor medida, son viejos conflictos derivados de períodos no lejanos de afirmación territorial. En mayor medida, son nuevos conflictos originados en el interẻs coincidente y natural de competir por un determinado mercado, por el control de recursos naturales, o por una influencia económica o política determinante dentro de la región. Como todo conflicto internacional a veces su manifestación es más aguda. Sólo la sangre fría y la visión de los verdaderos estadistas permite colocarlos en su lugar al insertarlos en contextos más amplios en que predominan intereses comunes y más esenciales.

Pero no sólo cabría preguntarse si estas situaciones conflictivas son evitables en la configuración de un sistema regional activo, sino también si no son signos saludables del dinamismo que han alcanzado las interacciones a nivel regional. La experiencia histórica a nivel interno y a nivel internacional, pareceria demostrar que un conflicto en sí mismo no es algo negativo. Lo negativo podría ser no reconocer su existencia pues ello esterilizaría la imaginación necesaria para anticiparlo y regularlo. Y la falta de capacidad para anticipar o regular conflictos es lo que sí podría conducir a manifestaciones negativas y extremas: el conflicto armado (¿no ocurrió ello en Centroamérica?).

En América Latina es entonces fundamental retener la idea de que 
el conflicto es un componente normal de la vicla de relación entre los países y que un proceso de integración no sólo no anula sino que aun puede incentivar los conflictos de intereses. Pero al mismo tiempo, cabe plantear la idea que un proceso de integración a la vez que desata nuevas fuerzas conflictivas permite encuadrar las viejas $y$ las nuevas en un marco de cooperación que permite su anticipación y su regulación. ¿No es acaso esta iclea la que subyace en todo el esfuerzo de integración europea desde que Jean Monnet redactara su célebre memorándum de mayo de 1950 que conduciría al Plan Schumann? Lo esencial en el plan Monnet-Schumann no es tanto el objetivo de la Federación Europea sino la idea de encuadrar el conflicto secular entre Francia y Alemania en el marco de un sistema europeo de cooperación que permitiera a la vieja Europa desempeñar un rol significativo en el ya previsible sistema internacional dominado por su entendimiento ruso-americano.

La integración económica y su capacidad de encuadramiento de situaciones conflictivas merece, por cierto, un análisis más profundo ante la emergencia visible de un sistema latinoamericano dinámico y competitivo que comienza a presentar algunas características similares a las que en otros subsistemas internacionales condujeron históricamente a la guerra. Negar este tema sería inclinarse por una visión ingenua o romántica de la realidad latinoamericana. Exagerarlo serfa inclinarse por un pesimismo estéril.

La distinción entre programa o proyecto de integración, como conjunto de objetivos y mecanismos que persiguen un modelo valorado de relaciones recíprocas, y que se formaliza en instrumentos jurídicos internacionales, y proceso o realidad de integración, concebido como el conjunto -sistemático o no- de acciones comunes estatales o privadas, orientadas a incrementar el grado de interacción entre los sistemas económicos de un grupo de países a fin de obtener un determinado grado de unidad interna $y / 0$ externa, puede ser útil para evaluar los progresos alcanzados en la llamada "integración latinoamericana".

Podría entonces afirmarse que los últimos años se han caracterizado más por una crisis de concepción de los programas o proyectos de integración (por ej. del Tratado de Montevideo, de la resolución 100, del informe de los cuatro, de la declaración de los Presidentes), que por una crisis die los procesos o realidades de integración, en los que se han manifestado avances sustanciales. Ellos se reflejan en el comercio, en empresas conjuntas, en la interacción a nivel empresarial, en el desarrollo de la infraestructura, en acciones comunes en el plano externo, en intercambios de efectos de demostración u otras formas de influencias, en el acrecentamiento del valor de la variable "latinoamericana" en las políticas externas y' aun internas, 
etc: Avances que deben medirse descle la perspectiva del punto de partida y' de lo factible y no descle la cle las metas formales y de lo deseable. La primera sería la del político. La segunda la del ideológico.

2. LOS REPLANTEOS A LOS PROYECTOS DE LA DÉCADA DEL CINCUENTA: LA VINGULAGIÓN DE LAS IDEAS DE DESARROLLO, INTEGRACIÓN Y DEPENDENGIA

Cuando en febrero de 1960 un grupo de países latinoamericanos firmó el Tratadó de Montevideo y se comprometió a perfeccionar, mediante un programa de liberación y otros instrumentos complementarios, el establecimiento de una zona de libre comercio y a perseverar en sus esfuerzos para arribar a la meta de un mercado común regional, el contexto mundial y regional en que actuaban y sus objetivos inmediatos exan profundamente distintos a los actuales.

En el plano mundial, sólo los más perspicaces vislumbraban en forma nítida el paso de una era de confrontación nuclear al de una confrontación industrial y tecnológica, en la que prevalece el multipolarismo y en la que se atenúa el conflicto ideológico con todo lo que ello significa para el margen de maniobra en el sistema internacional para los países periféricos. Las corporaciones internacionales existían, pero no se las percibía como actores transnacionales esenciales para comprender la trama interna de la vida política y económica mundial. La tecnología era producida, comercializada y consumida. Pero aún no se tenía conciencia de su carácter de mercancía, de las condiciones en que se la negocia, y del valor que tiene el poseer una capacidad propia en materia de creación y negociación de tecnología en función de una participación real en el juego del poder mundial. Los foros de participación internacional eran limitados y todavía no se había producido la innovación institucional que significa la creación de la UNCTAD.

En el plano regional, la intensidad de las interacciones económicas y políticas era reducida. Se limitaban, en el campo económico, al intercambio de productos primarios entre algunos pocos paises. Los países de la región exan compartimentos estancos, poco conocidos entre sí, que en muchos casos definían sus relaciones recíprocās en función de situaciones derivadas del siglo pasado. Sus mayores relaciones recíprocas se daban como consecuencia de su participación en el sistema interamericano que en aquel entonces comenzaba un 
proceso de cambio, en especial, por la creación del Banco Interamericano de Desarrollo.

En cuanto a los objetivos inmediatos que llevan al Tratado de Montevideo son conocidos. Estaban directamente vinculados al interés de los países del llamado "cono sur" por establecer un marco jurídico que les permitiera continuar, y eventualmente acrecentar, sus corrientes de comercio en términos preferenciales, y superar así el agotamiento y anacronismo relativo de los esquemas bilaterales. Por cierto que la idea del mercado regional estuvo presente en la negociación. Como se señaló antes, el preámbulo del Tratado y algunos artículos como el 54 asi lo atestiguan. Pero parece claro que esta idea se presentaba más bien como un objetivo de largo plazo, y que en todo caso no fue la razón principal que impulsó a la firma del Tratado.

En los años sesenta, entonces y al menos para los países de la ALALC, la idea de integración es percibida por los países en términos de comercio y de un posterior mercado común. La concepción prevalente es marcadamente economicista y en ella se refleja el pragmatismo de quienes interpretan intereses nacionales inmediatos $y$ en parte la nacionalidad de quienes se inspiran en los esquemas teóricos de la integración económica elaborados en Europa y los Estados Unidos. Ĺa integración es un medio - a veces sólo indirecto y hasta marginal- para maximizar bienestar. Poco tiene que ver con el juego del poder.2

Casi quince años después la idea de integración sigue vigente en América Latina. Lo que quizás ha cambiado es el contenido de la idea, la forma en que se concibe e imagina tal integración, su sentido como instrumento en un mundo profundamente distinto $y$ en proceso de cambio acelerado, los motivos por los cuales los países buscan desarrollar acciones conjuntas que impliquen unidad interna y unidad externa de Ia región o de parte de ella. La idea de integración está adquiriendo, en forma gradual pero segura, un claro contenido político, aun cuando planteada en términos económicos. No se la vincula sólo con comercio. Ni siquiera sólo con desarrollo. Se la vincula más bien con la creación de un entorno externo que permita acrecentar la participación real de los paises latinoamericanos en el sistema internacional. Se la vincula con la decisión de asegurar la viabilidad económica y política cle cada país de la región en un mundo en el que existe una marcada tendencia al congelamiento de la actual distribución del poder. Se la vincula, en la percepción más politizada, con el objetivo de ser actores y no meros espectadores de un proceso histórico apasionante. Se la vincula, por

- 2Of. PENA, Félix, "El Grupo Andino: un nuevo enfoque de la participación internacional de los paises en desarrollo", en Estudios Internacionales, No 29, 1973. 
fin, con la toma de conciencia de que es necesario acelerar cambios profundos en las estructuras políticas, sociales y económicas internas, por razones de justicia y como un medio de asegurar la supervivencia como unidades autónomas del sistema internacional. Las sociedades anacrónicas carecerían de la fuerza material y espiritual suficiente para evitar su marginación y eventual desaparición. La integración es un medio, y para algunos países, uno de los principales, a fin de maximizar poder en el plano internacional y de modernizar las estructuras sociales internas, lo que equivale por cierto a redistribuir poder.

Integración, desarrollo y participación real en el sistema internacional son tres ideas fuerza indisociables en la estrategia nacional de muchos países latinoamericanos. Lo pusieron de manifiesto los Ministros de Relaciones Exteriores del Grupo Andino en la reunión de 1973. Y sólo han reafirmado al más alto nivel político una relación que ha estado presente desde la Declaración de Bogotá en 1966. Está explícita en la propuesta de la SIECA para el perfeccionamiento y la reestructuración del Mercado Común Centroamericano, $y$ en el proceso de transformación de CARIFTA en CARICOM. Se manifiesta en más de un pronunciamiento oficial reciente de alto nivel.

El Grupo Andino ha sido el principal intento de operacionalizar la asociación de estas tres ideas en el marco de un programa multilateral coherente y de largo plazo. Contenido el programa en forma embrionaria en la Declaración de Bogotá, y luego en el mismo Acuerdo de Cartagena, se lo ha ido desarrollando empíricamente a través de decisiones de la Comisión, que como la del régimen común de inversión extranjera, la del primer programa sectorial de desarrollo industrial, la del programa de creación de tecnología, etc., han definido el perfil político y el modelo de desarrollo y de inserción internacional, que privilegian los países miembros. Las recientes definiciones en el plano financiero e institucional del programa de la Cuenca del Plata (reunión de Buenos Aires), los trabajos que han encarado el Comité de Alto Nivel en Centroamérica, y los primeros pasos de la Comunidad del Caribe, incluyendo sus estudios en materia de tratamiento a la inversión extranjera, parecen indicar que el replanteo de la concepción original de la integración latinoamericana está llegando a un punto culminante. Será quizás en las negociaciones colectivas que este año se realizarán en el marco de la ALALG, y en otras iniciativas que se han efectuado al más alto nivel político por parte de Perú y Venezuela, donde se manifestará la oportunidad de extender el replanteo al marco inicialmente establecido en 1960 por el Tratado de Monterideo. El que la oportu- 
nidad se manifieste no quiere decir que ella necesariamente se aproveche.

Quizá lo más interesante es observar, que en última instancia, la concepción misma de la integración y su formulación en programas multilaterales, se está aproximando a las realidades de las acciones integradoras de los últimos años en la región. Es decir, se está i.dentificando cada vez más con las múltiples acciones, que a nivel bilateral o multilateral, que en el plano subregional o en el de toda la región, que en forma global o sectorial, están desarrollando los países latinoamericanos a fin de organizar un subsistema económico y político internacional, en el que el área de la cooperación supere, neutralice o al menos encuadre, al área de conflicto, y que sea funcional a los objetivos de cambio interno y de incremento de participación real en el sistema internacional global. Parecería existir un menor apego a preconceptos $y$ a instrumentos muchas veces importados. No existe ya un respeto temeroso a una especie de ortodoxia integracionista que antes parecia ineludible. Por el contrario, se observa un saludable afán heterodoxo que estimula la audacia y la innovación y un cierto relevo generacional podría explicar el cambio. "Zona de Libre Comercio", "Unión Aduanera", "Programa de Liberación Comercial", "Mercado Común", "Libre circulación en factores", comienzan a ser observados con los ojos a veces poco respetuosos de quienes se sienten impulsados a probar, y aun a equivocarse usando medios propios.

Se puede afirmar que el contraste mayor entre la concepción de la integración, su formulación en un programa, y su operacionalización, tal como parecen darse en estos momentos con lo que ocurría en los años 60 es que en la actualidad en América Latina existe una experiencia en materia de integración. Experiencia que permite en cierta forma independizarse de las experiencias externas, que eran casi las únicas que existían en los 60. Experiencia que puede plantearse al menos en términos negativos. Es decir, de lo que los países latinoamericanos parecerian rechazax o no aceptar en el proceso de integración. Es posible afirmar, en efecto, que sobre la base de la experiencia de la década anterior los jaíses de la región no aceptan que sea suficiente la liberación del comercio como instrumento de integración económica; o que sea posible prescindir de mecanismos que creen la oportunildad de una participación real y equitativa de los países menores; o que sea admisible un mecanismo institucional que debilite la posibilidad cle que un país defienda su interés nacional en la elaboración de las decisiones conjuntas, pero que a la vez es factible encontrar un!mecanismo de concertación de intereses nacionales que salvaguarde a éstos permitiendo sin embargo la adopción de decisiones efectivas; o que no sea imaginable la compa . 
tibilización al menos en plazos cortos y medianos de un proceso de integración intenso entre países con sistemas económicos y políticos contrapuestos; o que sea posible disociar los objetivos e instrumentos de unidad interna entre un grupo de países con los que se refieren a la unidad externa; o que la participación en un proceso de integración excluya la posibilidad de desarrollar una estrategia internacional de apertura a todo tipo de países y a todo tipo de alianzas. En cada uno de estos puntos es posible citar experiencias concretas que explican la actitud generalizada al respecto.

Donde quizás haya todavía un mayor déficit es en la imaginación de fórmulas operativas que permitan concretar ideas aceptadas en principio. Pero aquí también puede detectarse un progreso significativo en la organización de técnicos capacitados en el sector público nacional dedicados a la formulación del interés nacional a través de propuestas destinadas a ser implementadas en marcos bilaterales o multilaterales, regionales o subregionales. En casi todos los países de la región se han establecido organismos nacionales de integración que al contar con la posibilidad de identificar y definir fórmulas satisfactorias al interés nacional introducen un elemento esencial -y que estaba ausente anteriormente -en el proceso de concertación de intereses en el plano multinacional. Quizás pueda señalarse un déficit en la capacidad de imaginación pragmática en los organismos internacionáles, en donde burocratización y rutina han sido a veces fuente de falta de dinamismo, y de capacidad de adaptación a las nuevas realidades.

3. SUBREGTONALIZACIÓN Y SECTORIALIZACIÓN EN EL ENFOQUE DE LA INTEGRAGIÓN REGIONAL: ¿SON COMPATIBLES GON UN PROYEGTO GLOBAL DE INTEGRAGIÓN LATINOAMERICANA?

Una de las características más notables de los replanteos que se han: efectuado en los programas de integración de la década del cincuenta, y en particulax en el de la ALALC, es el de la aceptación gradual de las estrategias subregionales y sectoriales como una forma de viabilizar y aun de acelerar la obtención del objetivo final de la integración global. Ambas son percibidas como vías complementarias para encarar la integración global a través de aproximaciones sucesivas e indirectas.

En particular, la idea de la subregionalización significa una reacción con respecto al enfoque original de la ALALC. Se volvió así a una vieja idea de colocar a los países medianos y menores en situa- 
ción previa de participar en condiciones dé igualdad en un proceso del cual también participarian los países'grandes de la región. La tradición subregional en realidad es más fuerte en América Latina que la regional. Los proyectos del siglo xix estuvieron concentrados en el área del Pacífico o en Centroamérica. El Caribe fue siempre una subregión. Aun en Ios trabajos pioneros de la CEPAL se plantearon ideas de subregionalización. La Carta de Quito de 1948 precede al proceso que llevaría a Montevideo.' En la reunión de Quitandinha de 1954, una resolución en la que habían trabajado actores de relevancia en el lanzamiento de la idea del Grupo Andino, plantea la aceptación de las aproximaciones subregionales. $Y$ aun 'en Montevideo estuvo presente la idea de la subregionalización. De allí que ella no fuera negada explícitamente en el Tratado y se abriera la puerta a la posterior resolución 222 de 1967 que permitió compatibilizar al Acuerdo de Cartagena con la ALALG.

La idea de la subregionalización tiene su fundamento económico en términos de equiparación de las posibilidades de aprovechamiento de las ventajas originadas en la formación del espacio económico regional y de evitar las distorsiones que de lo contrario se producirían a favor de las economías más clesarrolladas. Tiene todavía un mayor fundamento político en términos de acrecentamiento del poder de negociación de los países de "mercado insuficiente" y de "menor desarrollo" frente a los países "grandes" de la región.

También Ia idea de sectorialización se encuentra en el origen mismo del proceso de integración en los años cincuenta. Se expresa en el instrumento de los acuerdos de complementación industrial, no tanto en la forma en que ellos son utilizados en la. ALALC, sino en la forma en que ellos son concebidos en la CEPAL. Basta releer trabajos de la CEPAL y discursos de Prebisch en la época del Comité de Comercio para reencontrar el enfoque sectorial en toda su plenitud $^{3}$. El contraste también se observa en el cașo de la integración centroamericana con el régimen de industrias de integración, tal como fuera concebido originariamente y. luego formalizado. Cuando se formula el Acuerdo de Cartagena se acentúa la importancia de los programas sectoriales para la estrategia del desarrollo subregional.

En la práctica, la aproximación sectorial implica reconocer que no en todos los sectores de la actividad productiva es posible o es necesaria la integración. La posibilidad o la necesidad puede determinarse con criterios económicos o políticos. O ambos a la vez. Por ejemplo, dimensión de la demanda para encarax detexminada actividad, existencia de intereses creados (producción ya existente), o vinculación de la actividad respectiva a intereses políticos de valor

3Cf. entre otros, NACIONES UNDDAS, "Problemas actuales del comercio inter: latinoamericano"; E/GN. 12423, enero de 1957, p. 23. 
superior (seguridad nacional). A partir de dicho reconocimiento se actúa con criterio selectivo y en forma relativamerite programada y nuevamente en la programación se recurre tanto a criterios económicos como a criterios políticos.

En la instan̈cia actual del proceso de integración en América Latina no parece necesario cuestionar las ventajas de lás aproximaciones subregionales o sectoriales. 'Son hechos. Quizá más interesante resulta preguntarse si estos hechos son compatibles con la idea de la integración global de América Latina. Daría la impresión que esté interrogante formulado en relación a la idea de integración global tal como se planteaba, por ejemplo, en Punta del Este en 1967, podría carecer de importancia práctica. Es decir el inteirogante acerca de la compatibilidad de las estrategias subregionales y sectorialés con la idea de un Mercado Común Latinoamericano. Y ello debido a que la idea misma de un Mercado Común Latinoamericano, a imagen y semejanza de lo que formalmente debería ser el Mercado Común Europeo, comienza a desdibujarse como factor motor de las relaciones regionales lúego que en Asunción en el mismo año 1967 se constatara cuán lejos estaban los países de pensar seriamente en ella. Más sentido tiene el interrogante cuando la idea de la integración global, más que como una meta económica que adopte algunas de las formas clásicas derivadas de la teoría de integración económica, se plantèa como la expresión última de una identificación regional frente al resto del mundo. De tal manera, se la entiende como el rechazo de toda posibilidad que en su afán de desarrollo y de participación internacional los países de la régión puedan repetir la expèriencia históricá de otras regiones del mundo que lo hicieron a costa de la división $y$ del enfrentamiento riolento.

Subregionalización y sectorialización en la integración, implican enfóques parciales pero compatibles desde un punto de vista técnico. La sectorialización puede por ejemplo permitir la convergencia de ésquemàs subregionales en la medida en que tenga lugar entre países părticipantes de distintos agrupamientos (por ejemplo, participación del Mercado Común Centroamericano o del CARICOM en programas sectoriales del Grupó Ándino o con otros países de la ALALC). Desde el punto de vista político lo importante es que no impliquen una fragmentación del sistema régional en compartimentos estancos - contrapuestos. 'En tal sentido, no es tan importante definir dn esté momeinto la formá que adoptará en el largo plazo la'integración global. Lo importante es mantener como meta la idea de un sistema latinoamericano con objetivos, reglas de juego e instituciones comunes que sea un medio para la expresión nacional de sus componentes $y$ que se integre en forma diferenciada en el sistema internacional global. Así, ni la región negaría a la nación, ni impediría - por el. 
contrario facilitaría- una inserción ventajosa del conjunto de naciones en el conjunto internacional. Y para ello es necesario, en el corto plazo, facilitar el entrecruzamiento de los esquemas subregionales y sectoriales. Por el contrario, tratar en este momento de anticipar la forma que revistirá el proceso en su etapa final podría significar recaer en algunos exrores de la década del sesenta introduciendo elementos de rigidez ideológica en las relaciones interlatinoamericanas.

4. UNA TENSIÓN SERIA: LA INTEGRACIÓN COMO PLANTEO MIULTILATERAL $Y$ LA INTEGRAGIÓN COMO SUMA DE PLANTEOS BILATERALES.

¿ES POSIBLE CONCILIAR AMBOS PLANTEOS?

Aun aceptando o basándose a veces en la subregionalización y en la sectorialización, la concepción integxacionista de la CEPAL fue básicamente multilateral. Es decir, implicaba la participación conjunta de al menos varios países latinoamericanos sobre bases de no discriminación en el desarrollo del comercio y del espacio regional. También la tendencia predominante en ese periodo en el sistema internacional, en especial a partir del GAT $\Gamma$ y de su artículo 1, era hacia el multilateralismo. La "vía pragmática" de las relaciones económicas regionales, expresada en particular por los países del "cono sur", era en cambio básicamente bilateralista. Los entendimientos comerciales debían ser país por país. Cuanto más podrían llegar luego a su multilateralización como consecuencia de la aplicación de la cláusula de la nación más favorecida. En las negociaciones del Tratado de Montevideo era eso lo que se buscaba. Se reflejaba así la experiencia histórica de las relaciones regionales. Se llegó al multilateralismo, y se le dio forma de "zona de libre comercio" al instrumento elegido pues así lo exigía la compatibilización entre el acuerdo regional y los compromisos internacionales ya adquiridos o por ásumirse. No existía ni vocación ni experiencia multilateral. Y ello se reflejó tanto en las negociaciones como en la puesta en funcionamiento del Tratado y en la interpretación que originalmente se hizo de sus instrumentos.

Parece observarse ahora un retorno al bilateralismo. Ese retorno se observa no sólo en América Latina sino en todo el sistema internacional, particularmente luego de la crisis del petróleo. Los países aparecen como urgidos por concluir acuerdos comerciales, industriales y tecnológicos con otros países, a fin de asegurarse mercados y recursos. Es propio de períodos en que mercados y recursos comienzan a ser escasos. $Y$ por ello es fácil de comprender el interés na- 
cional envuelto en el retorno al bilateralismo. Como todos los países tienden a practicar el bilateralismo se debilita el condicionamiento internacional que antes impulsaba a una aceptación, aun contra voluntad, del multilateralismo. Es cierto que la política bilateral, país a país, siempre ha existido aun en el marco de políticas y acuerdos multilaterales. Nos estamos refiriendo, por tanto, a un retorno al bilateralismo como principio ordenador del comercio y de: las relaciones económicas entre naciones cuya expresión máxima podrían ser los acuerdos de trueque.

El bilateralismo puede ser discriminatorio. Si lo es, puede ser un camino directo a una escalada de confrontación económica entre los países del sistema internacional o de un subsistema parcial como el latinoamericano. A veces se ha denominado dicha confrontación "guerTa económica". Y a veces ha precedido las guerras en serio.

La integración como suma de planteos bilaterales podría llegax a ser inmanejable en el tiempo. Desde el punto de vista técnico y también desde el punto de vista político. Si es practicada por los países mayores podría evocar -quizás injustamente- los instrumentos clásicos de la relación entre grandes potencias y países periféricos. Incentivaría las desconfianzas y recelos, y aun las políticas pendulares de países beneficiarios del interés competitivo de los grandes.

Si la tendencia al bilateralismo es un hecho, en parte por el fracaso de los esquemas multilaterales o su inoperancia relativa, parece prioritario encontrar fórmulas pragmáticas que permitan conciliar-bilateralismo con multilateralismo. Dichas fórmulas serían más factibles en la medida que la subregionalización, la sectorialización, y el bilateralismo; sean expresamente incorporados en um planteo global sumamente flexible, que en sí mismo contendría el valor simbólico antes referido de rechazo a la idea de fragmentación del subsistema latinoamericano.

Para ello parece importante explorar Ia idea de un bilateralismo restringido a hipótesis previamente aceptadas y que sean compatibles con un multilateralismo final. Por ejemplo, la posibilidad de celebrar acuerdos sectoriales cerrados a pares de países pero por un número limitado de años, o con un sistema de apextura gradual. O la utilización del instrumento de "empresas conjuntas" binacionales a las que se atribuyan ventajas arancelarias o se les asegure el acceso privilegiado al mercado de sólo dos países, pero también por un periodo limitado. O acuerdos de cooperación tecnológica que sean parte de un programa más general de creación de tecnología a nivel subregional o regional. En cada una de estas fórmulas lo esencial no sería impedir el recurso a relaciones bilaterales particulares; sino exigir o estimular su apertura gradual al resto de los países.

La discriminación a evitar es sobre todo la del acceso a los mel- 
cados. Fórmulas como las sugeridas en el punto anterior pueden ser funcionales a clicho objetivo. El bilateralismo como política y como regulación de las relaciones económicas internacionales se expresa asimismo en concertaciones orientadas a la defensa de productos primarios en el exterior, a la explotación conjunta cle recursos naturales, al intercambio de materias primas por plantas industriales o por cooperación tecnológica, etc. Varios acuerdos han sido celebrados recientemente en América Latina entre pares de países y muchos de ellos son similares a los que se han celebrado entre paises productores y consumidores de petróleo o entre países con sistemas económicos diferentes. Las exigencias de un desarrollo solidario de la región justificarían que en ciertos casos sea recomendable que estos acuerclos bilaterales, aún no significando discriminación comercial, queden abiertos a la participación conjunta de varios países de la región.

\section{LA INTERACGIÓN ENTRE LA DIMENSIÓN INTERNA Y LA DIMENSIÓN EXTTERNA DE LA INTEGRACIÓN}

Resulta difícil imaginar un proceso de integración consensual sin una clara definición externa de quienes participan en el mismo. Quizás el elemento de diferenciación frente a terceros es el que más contribuye a consolidar la idea de grupo. Para ello es fundamental la percepción de un desafío externo que de una forma u otra cuestione la superviviencia de los componentes del grupo como unidades autónomas del sistema internacional. La percepción de un desafío externo - de distinta naturaleza por cierto- parece clara en los orígenes del Plan Schumann, en la superación de cada una de las etapas cruciales de la integración europea y en el caso del Grupo Andino. No lo es así en otros programas de integración, y en particular en la ALALG de los años 60. En el caso del Grupo Andino la definición externa originaria fue intralatinoamericana (reacción frente a los paises grandes de la ALALC). Una vez puesto en funcionamiento el Acuerdo de Cartagena, es en cambio la decisión 24 como respuesta a la penetración de las corporaciones transnacionales, la que contribuye a perfilar la personalidad internacional del Grupo. Dicha decisión fue elemento decisivo en la configuración de la imagen que el Grupo tenía de sí mismo. Ella define el modelo de inserción al sistema internacional que privilegian los países andinos. Como se sostuvo antes, tal definición se completa luego con otras decisiones importantes como la reciente sobre creación de tecnología, y con el com- 
portamiento conjunto del Grupo frente a los demás países de la región y a los países industrializados.

La valoración de los mercados nacionales ante terceros a través de un acuerdo de integración y de una política común en materia de transferencia de recursos productivos desde el exterior, hacen a la dimensión externa de un proceso de integración con bases regionales o subregionales. En otros casos, el aporte al sistema internacional que se intenta revalorar, es el de un recurso escaso y de importancia para los sistemas económicos de países industrializados. En ambas hipótesis, las acciones de integración -que por cierto en la segunda implican un grado menor de unidad interna- suponen un intento de acrecentar el poder de negociación internacional de un grupo de paises fiente tanto a los países altamente industrializados como a las corporaciones internacionales. ${ }^{4}$

Parece fundamental explorar más aún el tema de la dimensión externa de los procesos de integración de base geográfica, y compararla con la de los esquemas de integración funcional entre productores de materias primas. Daría la impresión que sólo en el primer caso, cuando en un grupo de países la acción conjunta se expresa tanto hacia el exterior como hacia el interior; es posible mantener en el tiempo una estrategia de acrecentamiento del poder de negociación internacional sin que se sufran los efectos de erosión y división producidos por la estrategia de respuesta de los paises grandes. En otros términos, si se visualiza tanto al Grupo Andino como a la OPEP, como instrumentos de acrecentamiento del poder de negociación internacional de países en desarrollo, la hipótesis sería que en el primer caso la acción sería menos espectacular que en el segundo pero quizás más efectiva y sólida en el largo plazo. El punto débil de la estrategia de integración en el campo de los productos básicos podría ser no tanto la capacidad de generar excedentes financieros como consecuencia del aumento de precios sino la capacidad para aprovecharlos en la creación de una base de sustentación de poder internacional a largo plazo. La combinación de una acción en el plano de los recursos primarios con una acción en el plano de la integración regional puede en cambio crear gradualmente las condiciones para que un conjunto de paises alcance la dimensión mínima requerida para una participación real en el sistema internacional.

Es interesante observar que dicha combinación se da en América Latina en el caso de los países productores de petróleo o de cobre en el Grupo Antino, o para poner otro ejemplo, en el de los produc-

\footnotetext{
*Cf. op. cit. en nota 2, y también del mismo autor, "North-South Relalions and Multinational Enterprises", informe presentado en la reunión sobre "A Dialogue on World Development", celebrada en marzo de 1974, bajo el patrocinio de Ja Charles F. Kettering Foundation y el Overseas Development Council.
} 
tores de bauxita en la Comunidad del Caribe. En la práctica, los países latinoamericanos se inclinarían a no concebir ambas estiategias como excluyentes, y a aprovechar el margen de maniobra que brinda el sistema internacional actual a través de su multipolarismo económico para perseguir simultáneamente objetivos de revalớización de mercados y de materias primas.

6. UN DESAFÍO PARA LA REGIÓN: DEFINIR UN PROYEGTO LATINOAMERICANO QUE PERMITA A GADA PAÍS EXPRESAR SU INDIVIDUALIDAD EN EL MARCO. DE UNA SOLIDARIDAD REGIONAL EFEGTIVA.

El mantenimiento de las identidades nàcionales parece ya indisociable de la idea de integración. Se está de vuelta del mito de los sesenta en el sentido que la integración suponía "supranacionalidad", entendida como negación de lo nacional a través de la disolución de los Estados naciones en una nueva comunidad política organizada. Si ello es claro, no lo es tanto el problema de la conciliación en la práctica de ambas realidades: la nacional y la regional. Una creación institucional gradual, basada en el aprendizaje progresivo del arte de la concertación de intereses y en el respeto creciente a reglas de derecho comunes, parece ser la única fórmula posible. Desde el punto de vista técnico lo es. Ello por cierto en la medida que cada Estado sepa cuál es su interés nacional.

Pero más difícil será aún demostrar que la integración habrá de dar lugar a una solidaridad regional efectiva. La prueba estará dada en los próximos años por la capacidad que demuestren los países latinoamericanos en acrecentar sus relaciones económicas reciprocas sin reeditar los esquemas de dominación propios de otros subsistemas económicos internacionales activos. En tal sentido, habrá que observar con àtención las pautas de comportamiento que sigan los paises mayores en sus relaciones con los de menor desarrollo de la región, y en particular, con aquéllos que les son contiguos. Muchas ideas han sido ya avanzadas en tal sentido y algunas han alcanzado estado de concreción. La puesta en común de recursos para el desarrollo de proyectos industriales o de infraestructura, la latinoamericanización gradual sobre bases multilaterales, de la cooperación técnica y financiera necesaria para el desarrollo de los paises menos industrializados, el desarrollo de programas multilaterales de creación de tecnologia, el reforzamiento de preferenciales arancelarias más favorables para los paises de menor desarrollo, son sólo algunos de los instrumentos funcionales a los objetivos de desarmollo solidario y de latinoameri- 
canización del sistema regional que han expresado en múltiples oportunidades y al más alto nivel los países de la región.

Quizás ha llegado el momento de reafirmar los objetivos reconocidos de la latinoamericanización del sistema regional sobre bases multilaterales y.de desarrollo solidario, en un acuerclo al más alto nivel, que sirva de encuadre para las acciones concretas de integración y de cooperación que se deseen encarar, sean ellas bilaterales o multilaterales, sectoriales o globales, regionales o subregionales, expresiones de unidad interna o de unidad externa.

El acuerdo se basaría en la convicción que en el futuro el actual período latinoamericano será considerado como algo original en la historia del hombre, no tanto en la medida en que se haya logrado una integración de las economías, o en la medida en que se haya logrado una alteración de las relaciones de dependencia, sino en la medicla en que se haya demostrado la viabilidad de un sistema de relaciones internacionales que posibilite el desarrollo de sociedades más justas, y que excluya la guerra como medio de solución de los conflictos y la dominación como elemento ineludible del diálogo entre grandes y chicos. Quizás el desafío es tanto más apasionante si se piensa que históricamente Europa fracasó en tal intento. 